\title{
Barriers and Enablers of Healthcare Providers to Deprescribe Cardiometabolic Medication in Older Patients: A Focus Group Study
}

\author{
Jamila Abou ${ }^{1}$ D $\cdot$ Stijn Crutzen ${ }^{2} \cdot$ Vashti Tromp $^{1} \cdot$ Mette Heringa $^{3,4} \cdot$ Rob Van Marum $^{5,6} \cdot$ Petra Elders $^{7} \cdot$ Katja Taxis $^{8}$. \\ Petra Denig ${ }^{2} \cdot$ Jacqueline Hugtenburg ${ }^{1}$
}

Accepted: 19 December 2021 / Published online: 21 February 2022

(c) The Author(s) 2022

\begin{abstract}
Introduction Benefits and risks of preventive medication change over time for ageing patients and deprescribing of medication may be needed. Deprescribing of cardiovascular and antidiabetic drugs can be challenging and is not widely implemented in daily practice.

Objective The aim of this study was to identify barriers and enablers of deprescribing cardiometabolic medication as seen by healthcare providers (HCPs) of different disciplines, and to explore their views on their specific roles in the process of deprescribing.

Methods Three focus groups with five general practitioners, eight pharmacists, three nurse practitioners, two geriatricians, and two elder care physicians were conducted in three cities in The Netherlands. Interviews were recorded and transcribed verbatim. Directed content analysis was performed on the basis of the Theoretical Domains Framework. Two researchers independently coded the data.

Results Most HCPs agreed that deprescribing of cardiometabolic medication is relevant but that barriers include lack of evidence and expertise, negative beliefs and fears, poor communication and collaboration between HCPs, and lack of resources. Having a guideline was considered an enabler for the process of deprescribing of cardiometabolic medication. Some HCPs feared the consequences of discontinuing cardiovascular or antidiabetic medication, while others were not motivated to deprescribe when the patients experienced no problems with their medication. HCPs of all disciplines stated that adequate patient communication and involving the patients and relatives in the decision making enables deprescribing. Barriers to deprescribing included the use of medication initiated by specialists, the poor exchange of information, and the amount of time it takes to deprescribe cardiometabolic medication. The HCPs were uncertain about each other's roles and responsibilities. A multidisciplinary approach including the pharmacist and nurse practitioner was seen as the best way to support the process of deprescribing and address barriers related to resources.

Conclusion HCPs recognized the importance of deprescribing cardiometabolic medication as a medical decision that can only be made in close cooperation with the patient. To successfully accomplish the process of deprescribing they strongly recommended a multidisciplinary approach.
\end{abstract}

\section{Introduction}

Considerable efforts have been made to optimize the quality of the medication used by older patients with multimorbidity and polypharmacy [1]. Polypharmacy, commonly defined as the chronic use of five or more medications [2], may lead to more falls, hospitalizations, lower health-related quality

Jamila Abou

j.abou@amsterdamumc.nl

Extended author information available on the last page of the article of life, higher mortality and increased healthcare costs [3]. In older or frail patients with type 2 diabetes and/or cardiovascular disease, overtreatment with cardiometabolic medication has repeatedly been identified [4] with the risk of hypoglycemia, hypotension and muscle pain $[5,6]$. Agerelated physiological changes, such as impairment of renal and/or hepatic function, contribute to the increased risk of adverse drug events in this population. In addition, the long-term benefits and risks of many cardiovascular medications are unknown in older patients with multimorbidity [7]. Patients with limited life expectancy or with a life-limiting 


\section{Key Points}

Deprescribing of cardiometabolic drugs was deemed relevant by healthcare providers from all disciplines but its implementation needs further support.

Not all healthcare professionals have adopted a proactive deprescribing approach, which is needed to prevent adverse drug events in older and frail patients.

Good communication with patients and relatives was considered essential for deprescribing cardiometabolic medication, with some healthcare providers needing training to improve their skills to do so.

A multidisciplinary approach with agreements on information exchange and task delegation can be recommended given the perceived barriers and enablers for deprescribing.

disease have less opportunity for the long-term benefits of these drugs.

Increased frailty, a decline in cognitive function, and a decreased ability to manage daily living activities may require deprescribing of cardiometabolic drugs no longer considered sensible or safe $[8,9]$. The focus of the medication optimization process in older and frail patients has thus shifted towards deprescribing of medication and ways to effectively apply this concept in daily practice [10-12].

Deprescribing comprises the systematic reduction or withdrawal of medication that may cause harm or no longer contributes to an individuals' health status [13, 14]. Deprescribing of glucose-lowering medication in patients with comorbidity, frailty, hypoglycemic risk, or a limited life expectancy seems feasible and safe $[8,15,16]$. Furthermore, deprescribing of antihypertensive medication in frail older patients seems appropriate and feasible [7, 17]. In recent years, recommendations have been made to deprescribe cardiometabolic medication in older patients [7, 8, 18]. Nevertheless, deprescribing of cardiometabolic medication can be challenging for healthcare providers (HCPs) and is not yet widely implemented in daily practice $[4,12,15,19]$.

Several studies among HCPs have identified barriers to deprescribing as mentioned by HCPs, such as a lack of evidence that discontinuation would be more beneficial than continuation, uncertainty and fear of negative consequences, unwillingness of patients and relatives, and the lack of time or support to perform deprescribing [20-24]. These studies have explored barriers and enablers towards deprescribing in general, whereas such factors may differ for different medication classes. Moreover, the majority of the studies focused on the perspectives of clinicians. A study also including pharmacists illustrated that they often perceived different barriers towards deprescribing than general practitioners (GPs) [21]. Another study showed that the factors seen as important for deprescribing differed between GPs, community pharmacists (CPs) and nurses [25]. One study comparing HCP perspectives regarding deprescribing of cardiovascular medication concluded that there were substantial differences between medical specialties in both the reasons for deprescribing and the medication to deprescribe [26].

Since optimizing medication in older people using cardiometabolic medication in primary care often requires a multidisciplinary approach, it is relevant to explore the barriers and enablers seen by various HCPs involved, including GPs, nurses practitioners (NPs), CPs, geriatricians (GEs) and specialists in elderly care (SEs). The concept of pharmaceutical care and the role of different HCPs may differ in different countries. In The Netherlands, the CP is responsible for offering adequate pharmaceutical care and has a significant role in medication management in primary care. Given these tasks, the CP has frequent contact with other members of the healthcare team $[27,28]$. A model of having clinical pharmacists to provide pharmaceutical care is not yet implemented in Dutch primary care.

For studying barriers and enablers, the Theoretical Domains Framework (TDF) was considered appropriate [29]. This framework consists of 12 domains based on theoretical models or constructs that help determine the barriers and facilitators of implementing new behavior, for example related to healthcare practice.

The aim of the study was to identify barriers and enablers of deprescribing cardiometabolic medication as experienced by different disciplines of HCPs, and explore HCP views on their specific roles in the process of deprescribing.

\section{Methods}

\subsection{Study Design}

A qualitative study was conducted using a semi-structured interview format with three focus groups, each including a mix of HCPs, including GPs, CPs, NPs, SEs, and GEs.

\subsection{Setting}

In The Netherlands, GPs provide care for most patients with diabetes and/or cardiovascular diseases. NPs assist GPs in monitoring these patients and support them in using their cardiometabolic medication; NPs may suggest GPs to adjust medication regimens. CPs, together with GPs, have a leading role in conducting periodic clinical medication reviews to optimize the medication of older people with polypharmacy. SEs are mostly involved in the care of elderly patients in 
chronic care settings, such as nursing homes, and often have an advisory role in local primary care networks. GEs are part of secondary care. They are involved in the treatment of hospitalized elderly patients and the supervision of care of more complex elderly patients in primary care.

\subsection{Recruitment}

A purposive sampling technique was used to recruit participants. HCPs with at least 2 years of experience with care for older patients with chronic diseases in three large cities across The Netherlands were invited to participate by email. Some HCPs were selected from the researchers' network while others were selected by searching the internet for primary care health centers and hospitals providing contact emails. When HCPs expressed they were not able or willing to participate, they were asked to suggest others who might be able to participate. In total, around $30 \mathrm{CPs}, 10 \mathrm{GPs}, 7$ NPs, 5 GEs and 5 SEs were contacted. The aim was to invite at least twice as many GPs and CPs than members from the other disciplines, given their leading role in the conduct of clinical medication reviews for older people with cardiovascular and antidiabetic drugs in The Netherlands. One of the researchers (JA) contacted the HCPs by telephone if they did not respond to the email. Written informed consent was obtained from participants prior to each focus group discussion.

\subsection{Topic List}

The TDF was used to create a topic list on the basis of existing literature on barriers to and enablers of deprescribing [20-24]. The TDF consists of 12 domains describing important factors underlying behavioral change and implementation issues: knowledge, skills, social/professional identity, beliefs about capabilities, beliefs about consequences, motivation and goals, memory attention and decision processes, environmental context and resources, social influences, emotion, behavioral regulation, and nature of the behaviors [29]. Probing questions with several subtopics addressing TDF domains were phrased to explore the views of HCPs on the process of deprescribing and its implementation in daily practice. The topic list was pilot-tested with a $\mathrm{CP}$ and a GP, and subsequently adapted to obtain more in-depth information and to stimulate discussion. The final topic list started with a general question about the participants' ideas around deprescribing followed by eight probing questions with subtopics related to the 12 TDF domains, and ended with an open question asking for any additional aspects that had not been covered in the discussion.

\subsection{Data Collection}

Three focus group discussions of 90-100 min duration were held between December 2018 and February 2019-one in a conference center and two in a medical health care center. The spoken language was Dutch and the sessions were audiotaped with permission. The group discussions were moderated by PD (PharmD, PhD, Professor at a medical faculty), assisted by a junior researcher (JA, PharmD) who made notes in addition to the audiorecording. No other nonparticipants were present. PD has received interview training and is experienced with moderating focus group interviews with HCPs. She had no prior relationship with the participants nor were the participants informed about the interests of the interviewer. As a member of the research team, PD was involved in developing the topic list and had knowledge about previous research conducted in this area.

\subsection{Data Analysis}

Directed content analysis was performed on the basis of the TDF. Audiotapes were transcribed verbatim and the transcript was checked for accuracy by a member of the research team, making use of the notes when needed (JA). Transcripts were not checked by the participants. Two researchers (JA, VT) separately coded transcripts using Atlas Ti version 7.0. Thematic coding was applied to identify data related to barriers, enablers, and the roles of HCPs towards deprescribing. The domains of the TDF were used to categorize subthemes of barriers and enablers. The coding was discussed and any discrepancies were further discussed until consensus between the two researchers was reached. The participants were not asked to provide feedback. Quotes were selected for the manuscript to illustrate the themes. The specific barriers and enablers within the TDF domains were then grouped in overarching themes as created by the researchers after reviewing all data. Interpretations and grouping of data were made under the supervision of two senior researchers $(\mathrm{JH}$, PharmD, PhD; PD, PharmD, PhD). After the third focus group meeting, four researchers (JA, VT, JH, PD) discussed whether saturation had occurred by reflecting on key themes identified in each focus group.

\subsection{Ethical Approval and Check of Reporting}

This study was approved by the Medical Ethics Committee of the Amsterdam UMC, location VUmc (FWA00017598). It was based on generally accepted ethical principles, such as the right to refuse to participate in the study and the right of participants to withdraw at any time, as well as respect for all participants and the protection of their privacy. 
Table 1 Setting and focus group participants

\begin{tabular}{llll}
\hline Setting & $\begin{array}{l}\text { Focus group 1 } \\
\text { Health care center }\end{array}$ & $\begin{array}{l}\text { Focus group 2 } \\
\text { Health care center }\end{array}$ & $\begin{array}{l}\text { Focus group 3 } \\
\text { Conference center }\end{array}$ \\
\hline Total $(n)$ & 10 & 5 & 5 \\
Male $(n)$ & 4 & 0 & 2 \\
General practitioner $(n)$ & 3 & 0 & 2 \\
Community pharmacist $(n)$ & 3 & 2 & 3 \\
Nurse practitioner $(n)$ & 1 & 2 & 0 \\
Geriatrician $(n)$ & 1 & 1 & 0 \\
Specialist elderly care $(n)$ & 2 & 0 & 0 \\
\end{tabular}

The Consolidated Criteria for Reporting Qualitative Research (COREQ-32) list was used to check the reporting of important aspects of the research team, study design, analysis, and findings (see electronic supplementary material).

\section{Results}

\subsection{Participants}

Twenty HCPs participated in three focus groups, including five GPs, eight CPs, three NPs, two GEs and two SEs (Table 1). All HCPs had more than 5 years of work experience. GPs and CPs put forth the most barriers and enablers. Four overarching themes related to the implementation of deprescribing cardiometabolic medication emerged from the three group discussions. First, the need for sufficient evidence and expertise to conduct deprescribing, followed by the beliefs and fears of HCPs and others involved that may impede or promote deprescribing, and professional collaboration between the HCPs involved in the management of diabetes and cardiovascular diseases. Finally, the context and resources that can hinder or support the process of deprescribing. In each of the themes, two or three underlying TDF domains with sometimes overlapping or related statements were clustered.

\subsection{Evidence and Expertise}

HCPs discussed the knowledge and skills they considered important for initiating deprescribing of cardiometabolic medication. Overall, they mentioned more enablers than barriers on these domains, and indicated that sufficient evidence was needed about the effects of deprescribing as well as continuation of certain drugs. Most CPs and GPs wanted to have sufficient evidence in support of their recommendations before discussing deprescribing with patients, relatives or other HCPs. CPs mentioned that a respected national or international guideline recommending deprescribing of cardiometabolic medication would be very helpful to support their actions. On the other hand, HCPs from all disciplines indicated that the lack of evidence and information about the benefits and risks of deprescribing restricted their conduct of such deprescribing. Several GPs and CPs expressed that guidelines for the treatment of diabetes and cardiovascular risk management were not sufficiently clear about deprescribing.

- 'You need such an instrument, because if I stop this (a cardiometabolic) medicine, I have no idea what the risks are for the individual patient.' (GP3.2, B1).

Some GPs and CPs remarked that they sometimes had insufficient knowledge about novel cardiovascular or diabetes medication. This prevented them from adjusting certain treatment regimens.

- 'These newer drugs (for diabetes and cardiovascular diseases) change so rapidly. Which combinations are allowed or not?' (CP3.2, B3).

HCPs from all disciplines acknowledged that there is also insufficient evidence about the benefits of continuing preventive cardiometabolic medication in elderly or frail patients, and that the balance between the advantages and disadvantages of such treatment changes with the aging of the patient. In this context, the lack of evidence for continuation was considered an enabler of deprescribing. Most HCPs agreed that strict control of blood pressure and glucose levels is no longer required in elderly patients, and that given the increased risks for older patients, deprescribing of certain cardiometabolic medication is therefore an option.

- 'There are the new guideline targets and there you have 70-plus and more than 10 years diabetes, that you have to use different targets.' (NP2.1, E5).

Several HCPs expressed that deprescribing is possible when conditions are met regarding their expertise and skills. 
- 'I would not say that it is an impossible task but you just need [...] to feel that you have enough expertise that you are able, can, dare to stop.' (GP1.1, E8).

Several HCPs expressed that they found it difficult to talk with patients and their relatives about deprescribing. There were also HCPs who believed that they had sufficient expertise and skills to engage in deprescribing. Some stated that having more knowledge about deprescribing would contribute to better communication. It was also mentioned that more knowledge on how to teach elderly patients a healthy lifestyle could support the process of deprescribing.

- 'I agree with that (difficult conversation with patients). You have to have a good story for this, not giving up on somebody. Recently, I had a lady that did not want it. Whereas she used a lot of unnecessary drugs.' (CP3.2, B4).

- 'Better communication skills, for me, I have to do it (for the conversation with patients on deprescribing).' (GP3.2, B4).

One NP said that lack of knowledge might be a bigger issue for NPs compared with GPs and CPs. On the other hand, another NP indicated that they have the experience and skills to gain the trust of patients. Skills on how and when to taper, monitor or re-initiate antihypertensive medication and glucose-lowering medication were mentioned as enablers by HCPs from other disciplines. Some HCPs found it difficult, or were not motivated, to start deprescribing when there seemed to be no problems with the medication (Table 2).

\subsection{Beliefs and Fears}

HCPs from all disciplines reported being motivated to deprescribe cardiometabolic medication that is no longer needed or is potentially harmful. Some GPs mentioned that they were not motivated to deprescribe medication in relatively fit older people or in people not experiencing any problems.

- 'With diabetes, I would not stop if somebody of 80 is fit [...]. I would not think of stopping such medication if somebody still has 3 years to live.' (GP3.2, B8).

- 'I know that that stopping of medication is better than simply continuing it, that is my conviction.' (GE1.1, E14).

Uncertainty or fear about the consequences of deprescribing were seen as a barrier. For several HCPs, the potential negative consequences of deprescribing weighed heavier than the potential negative consequences of continuing treatment.
- 'It is easier to say I do nothing because then [...] I am not the one who takes the risk.' (GE1.1, B6).

The opportunity to restart medication in case of negative consequences was considered an enabler.

- 'If we deprescribe an antihypertensive agent and it the blood pressure) becomes too high, we can always adjust it again.' (GP3.2, E12).

HCPs were apprehensive about reactions from relatives who fear that treatment discontinuation of cardiometabolic medication will lead to disease deterioration. This could be an argument against discontinuation. CPs in particular had experienced negative reactions from GPs or patients when they recommended deprescribing a certain medication.

- There are people where you have changed something (in the past), which did not go well. In that case, the GP and the patient do not want to change it (the medication), ever [...]. This can be something that happened when they were 40 (years of age) and now they are 70 years.' (CP2.2, B11, B13).

- 'I often experienced that they are afraid of any changes in (their parents') medication, because this may not go well for their father or mother.' (CP2.1, B11).

According to most HCPs, the preferences of the patient are key in the process of deprescribing, emphasizing the importance of good communication with patients and relatives. Several HCPs indicated that when acting in this manner, there were hardly any negative reactions from relatives when suggesting to deprescribe medication. One SE emphasized that patients and relatives should be involved from the start in making shared decisions on their medication.

- 'If you involve the family from the first step [...], take them with you in the whole process [...], then you will have them on our side and everybody will be happy with that advice (to deprescribe).' (SE1.1, E15).

Several GPs felt uncomfortable changing or deprescribing specialist-initiated medication, especially when patients placed high value on this prescriber's judgment, and also when they believed that the specialists would not be willing to stop a certain medication.

- 'You often hear patients saying, the cardiologist has said that I have to use (the medication) my whole life [...]. You cannot touch it.' (GP1.1, B14).

- 'For cardiologists, Ascal is sacred. Also, neurologists, it is just sacred.' (GP1.2, B14). 


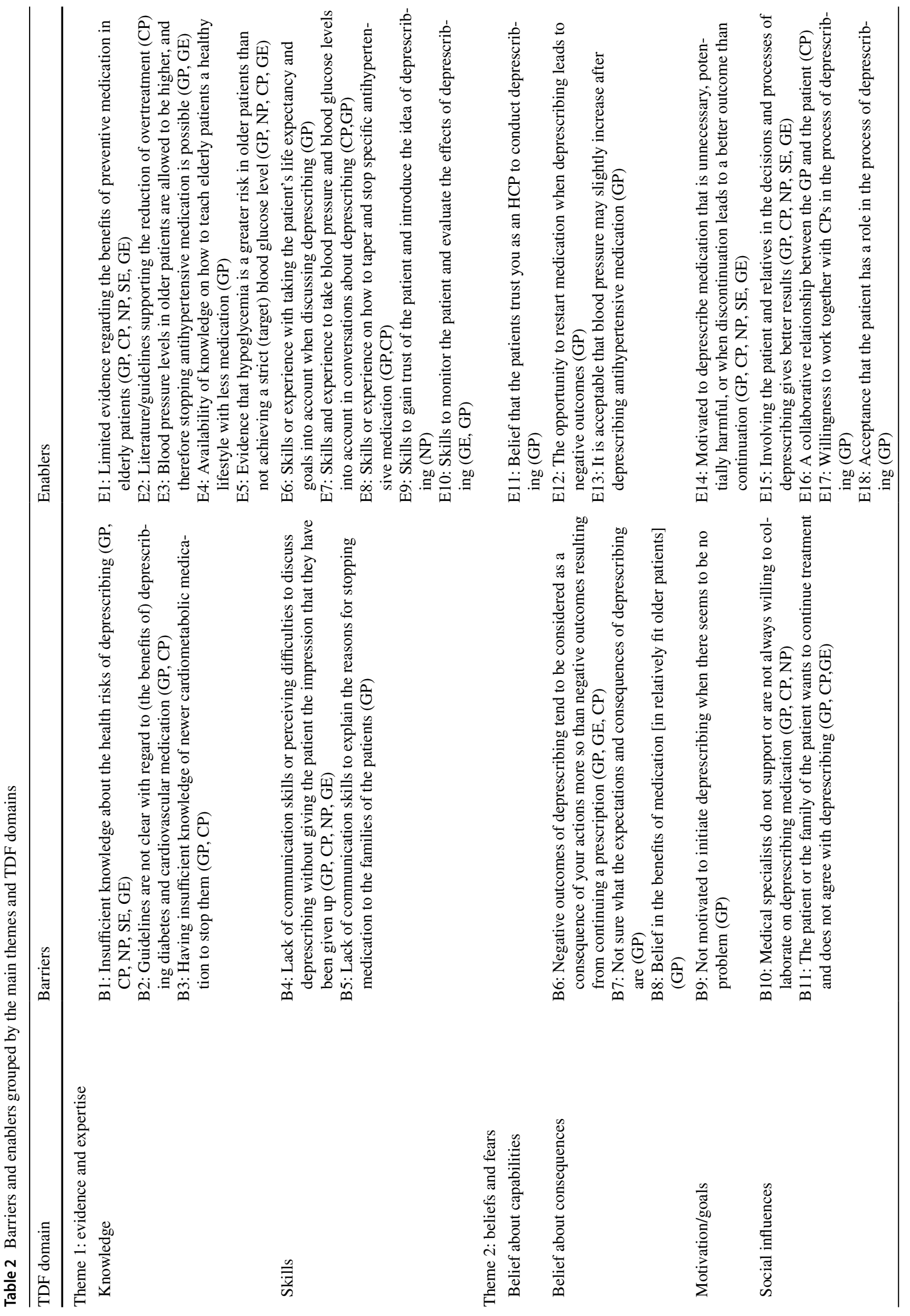




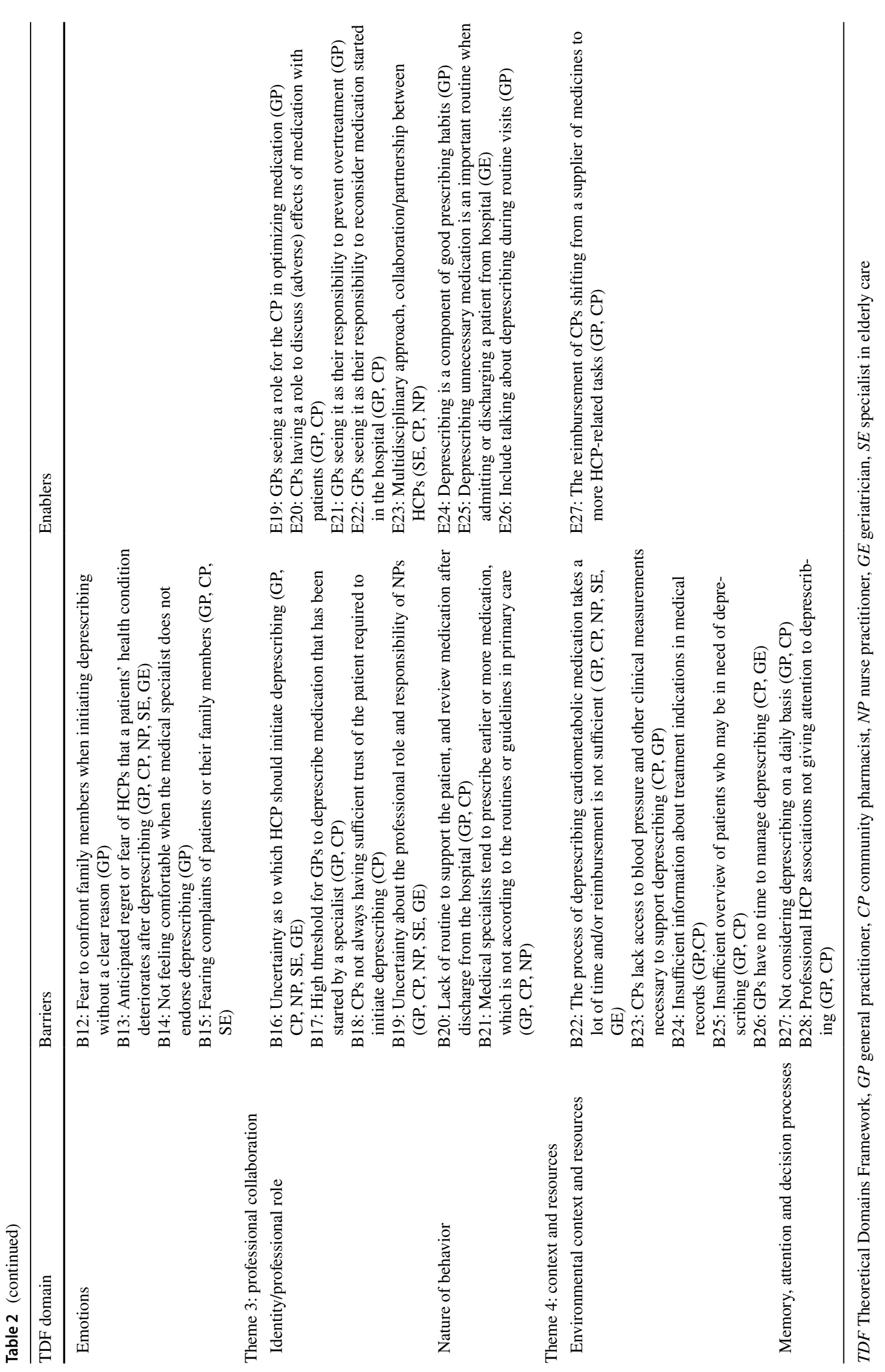




\subsection{Professional Collaboration}

Professional collaboration was an important theme for implementing deprescribing as part of routine care. For many CPs, sufficient multidisciplinary collaboration was an enabler for deprescribing, but an SE also mentioned the advantage of a multidisciplinary approach. One NP stated this is essential for HCPs not having prescribing authority. CPs mentioned that they are used to giving recommendations on deprescribing when they conduct clinical medication reviews together with GPs. Several GPs and CPs stated that they were not only willing but also found it a necessity to work together on deprescribing.

- 'Indeed, I think that we-being different HCPs-can complement each other.' (SE 1.1, E23).

- '(There are) a lot of interactions of medication that I don't know about, so I appreciate the input of the CP, especially in a medication review.' (GP3.1, E23).

- 'Given the complexity of these medications, the amount of possible inaccuracies in medical files, I see an important role for the CP.' (GP3.2, E23).

There was some uncertainty about who should take action.

- 'What I find very difficult is, is the responsibility only with me as GP or with the CP? The patient has no idea what the choice is. They want to stop.' (GP3.1,B16).

Aspects of task delegation were mentioned as enablers for deprescribing. CPs mentioned that they talk with patients about what they want, for example less medication or less adverse effects, as part of the medication reviews. On the other hand, CPs may not have sufficient trust of the patient to initiate deprescribing. One GP mentioned that the NP could start the conversation with patients, and the SE or CP could support the process of deprescribing further.

- It is the question who has the first conversation with the patient. I do not know how you (NP) think about this but I can imagine that the NP does this when the time is there and then involves the other professionals-either the specialist in elderly care when it is too complicated or the pharmacist to explain it further.' (GP1.1, E19, E23).

- 'It is nice to discuss this together, that you are on the same page. We have our geriatric meetings, where the pharmacist also joins us. Yes, you tell the same thing, why something can be stopped'. (SE1.1, E23).

GEs and SEs were accustomed to discussing deprescribing of preventive cardiometabolic medication and felt no obstacles in contacting GPs to discuss deprescribing in patients they both cared for. A GE stated that in the transition from hospital to outpatient care, more attention may be needed to inform the GPs and CPs about medication that has been stopped.

- '(We should) have a better transfer (from the hospital) to primary care, to the GP and the CP and the patient [...]. There (in the hospital) is the trend of giving a lot of information in a short period [...], but people afterwards do not remember it well (information about medication changes).' (GE 2.1, E25).

GPs felt burdened when they wanted to discontinue medication that had been started by a specialist. Complex relationships with other HCPs sometimes made it easier to just continue a prescription instead of deprescribing. Some, on the other hand, did feel responsible to take action.

- 'If there has been no information transfer about the initial indication for a medication, I would - also as a GPbe afraid to just stop it.' (CP1.3, B17).

- 'Yes, exactly (a cardiologist is top level) and you are just GP.' (GP1.1, B17).

- Yes, it is a duty to prescribe correctly and if it (a medicine) is not necessary, then you must take action.' (GP1.3, E21).

\subsection{Context and Resources}

Mostly barriers of deprescribing cardiometabolic medication were mentioned with regard to environmental factors and resources. It was mentioned that deprescribing of these drugs takes a lot of time. Some GPs indicated that there was insufficient attention and practical support from their professional association for deprescribing.

- 'You cannot stop (cardiovascular medication) at once, you often have to take several steps, and those steps take a lot of effort [...]. A GP actually may not have sufficient time for this.' (GE1.1, B22, B26).

CPs and GPs in particular expressed that a lack of information about, for instance, the indication of a specific medication or lack of access to clinical information could also be barriers for deprescribing.

Although HCPs from all disciplines mentioned insufficient reimbursement as a barrier, it was mentioned that for CPs, deprescribing may actually lead to a loss of income because they get reimbursed for dispensing medication. One CP mentioned that things may be changing and that she hoped that pharmacists in the future will be reimbursed for activities such as preventing harm from medication. 
- 'This costs me a lot of time [...]. I think it is my responsibility [...] but it does not help because I get less and less financial compensation from the insurance companies.' (CP 2.1, B22).

- 'I think that a lot will change in the pharmacy world, I hope that we do not have to think it that way anymore (worry about getting less income when you stop medication).' (CP3.2, E27).

\section{Discussion}

\subsection{Summary}

Four overarching themes were distinguished regarding the barriers and enablers of deprescribing cardiometabolic medication that were not restricted to a specific HCP discipline: evidence and expertise, beliefs and fears, professional collaboration, and context and resources. Most HCPs agreed that in older and frail patients, strict control of glucose levels and blood pressure may be more harmful than beneficial. This would clearly justify deprescribing of certain cardiometabolic drugs. In spite of this, some wanted more evidence and guidance to support deprescribing of these drugs. Several HCPs expressed that better communication skills to discuss deprescribing with patients and their relatives would be helpful. Previous negative experiences and uncertainty about the outcomes were barriers to initiate deprescribing. Some HCPs preferred to continue current treatment rather than to deprescribe medication. GPs in particular were less motivated to discontinue medication that had been initiated by a specialist or when the patient did not experience any problems. Expected resistance or fears of patients and relatives with regard to deprescribing were mentioned as barriers by several HCPs. Trust of the patient and good communication with patients and relatives was seen as key to their willingness to consent to the deprescribing of medication. Multidisciplinary collaboration was considered an important enabler to deprescribe, but our study showed that it was not yet clear who should do what in the process of deprescribing. Insufficient sharing of information was a barrier particularly indicated by CPs and GPs. Lack of resources and reimbursement was perceived as a barrier by most HCPs.

\subsection{Comparison with the Literature}

HCP barriers and enablers of deprescribing have previously been identified, mostly from the GP perspective, and are related to deprescribing of medication in general [20-24]. We included HCPs from different disciplines and found that the overarching themes were shared by all disciplines. However, at a lower level, there were differences between primary and secondary care HCPs as well as between physicians and other disciplines. By focusing on cardiometabolic medication instead of medication in general, we identified several medication-specific issues.

Nevertheless, there are a number of general barriers mentioned by all disciplines that seem relevant regardless of the medication considered. These include a lack of evidence and guidance on when deprescribing is beneficial and safe, a lack of collaboration and communication between HCPs, and a lack of time and resources to conduct deprescribing. Most of these general barriers are predominantly at the organizational and interpersonal levels, as described in the socioecological model used in a recent review of barriers and enablers to deprescribing in primary care [23]. The socioecological model is a theory-based framework hierarchically distinguishing barriers and enablers at the individual, interpersonal, organizational and cultural (i.e. society) levels, and can be used to better understand the complexity of inter-related barriers and enablers within the multiple levels of a social system, such as a healthcare system. In particular, this can be barriers and enablers at the cultural, organizational, interpersonal and individual level. There were also general enablers at these levels, which are not linked to specific drugs and perceived by all disciplines, such as enhanced multidisciplinary collaboration and task delegation. It should be noted that these barriers and enablers may differ depending on the context and healthcare system. For example, there may be differences in the role of the GPs, in particular whether or not GPs have a gatekeeper position [12,30], and in the relationship of GPs and CPs with community- and hospital-based specialists, and with it, their accessibility and willingness to collaborate $[12,31]$.

HCPs from all disciplines recognized that other disciplines could or should have a role in deprescribing. There was however no general consensus on who should initiate deprescribing. Taking responsibility and starting the process of deprescribing seems crucial [32]. The physicians in our study mentioned their responsibility to reconsider prescribed medication and prevent overtreatment in older people, but at the same time they indicated that CPs and NPs are relevant to support the process of deprescribing. The need for task delegation and the value of CPs and NPs has been stressed previously $[6,12]$. NPs in our study believed that they could contribute by suggesting the deprescribing of medications to patients but that they lack the knowledge required to make specific medication or patient-related judgments. This aligns with a previous finding where nurses ranked 'GP receptivity to deprescribing' and their own 'ability to advocate for the patient' as the most important factors [25]. CPs in our study considered a role for themselves in various parts of the deprescribing process, including conversations about deprescribing and guidance on how to taper and stop specific medications. In Dutch primary care, as in other countries, a collaboration between GPs and CPs to optimize the 
medication of older patients with polypharmacy already exists. Pharmacist-led clinical medication reviews in such patients may help to reduce the number of drugs used by patients [33, 34].

Regarding cardiometabolic medication, a lack of knowledge or expertise was perceived by some primary care HCPs. GPs and CPs in particular mentioned a lack of clear recommendations in guidelines on this topic as a barrier. This made them uncertain about deprescribing specific cardiometabolic drugs. It has previously been described that GPs and CPs can perceive difficulties in dealing with uncertainty, and in the case of specific medication, doubts about what is best for the patient [21]. Some drugs were considered 'easier' options for deprescribing because of greater perceived certainty that they were potentially harmful or no longer needed [21]. Enablers mentioned by HCPs from all disciplines included a lack of evidence that continuation of cardiometabolic medication would be beneficial and the knowledge that adverse effects of certain drugs become more problematic in older patients. They explicitly mentioned the unfavorable benefit-risk balance for antidiabetic drugs in older patients as an enabler. Some also mentioned that the higher tolerated blood pressure levels in older patients support deprescribing. On the other hand, some GPs were not motivated to initiate deprescribing of cardiometabolic medication in cases where patients were relatively fit and did not have problems with their medication. It seems that these HCPs had not adopted a proactive deprescribing approach, which is needed to prevent adverse drug events to occur [35]. This is in line with a previous study describing specialists' perspectives on deprescribing cardiovascular medications in older adults, where adverse drug events were the main reason to consider deprescribing [26]. Proactive deprescribing of cardiovascular medication for a hypothetical patient with no specific concerns was relatively low, ranging from $23 \%$ of cardiologists to $41 \%$ of GEs [26]. It was also found that GPs may feel more comfortable about discontinuing drugs used for symptomatic relief depending on the patient's need than to discontinue preventive medication, although these opinions also varied between GPs [36].

More in general and anticipated regret and the feeling that you are more to blame for the negative consequences of deprescribing in comparison with the potential harm of continuing medication were mentioned by HCPs from all disciplines. To overcome this barrier, a good relationship with patients, and involving patients and their relatives in the decision-making process, were mentioned, both in our study and in other studies [20-24]. Regarding the perceived trust of patients and their relatives, there were differences between HCPs from different disciplines. As observed previously, GPs experienced less barriers regarding this trust in comparison with CPs [21]. In our study, NPs also expected that they were able to gain the required trust of patients.
HCPs from all disciplines mentioned that a lack of communication skills is a major barrier and that the ability to communicate with patients and their relatives is highly important, as was concluded in previous studies [22, 23]. GPs and CPs in our study particularly mentioned the ability to talk about life expectancy and being able to take blood pressure and blood glucose levels into account in the conversations as enablers. All primary care HCPs experienced difficulties regarding deprescribing in case medical specialists were involved, which has been previously noted in several studies among GPs [23]. In our study, GEs and SEs were not concerned about deprescribing medication initiated by other clinicians. This contrasts with a recent study conducted in the US, where it was found that GEs, general internists and cardiologists were concerned about interfering with other clinicians' treatment plans [26].

\subsection{Strengths and Limitations}

By including HCPs from different disciplines, the themes that emerged were not restricted to the perspective of a specific HCP and the different views regarding their roles in the conduct of deprescribing could be explored. Furthermore, by focusing on deprescribing of cardiometabolic medication, we could explore medication-specific beliefs and attitudes. According to the principles of social psychology, these are expected to be closer related to actual decisions as to whether or not to deprescribe such medication [37]. We used the TDF to develop the topic list and guide the analysis, thereby covering all topics considered relevant for implementation research.

A limitation of this study lies in our sampling strategy, resulting in small numbers of NPs, GEs and SEs. In general, one could expect that HCPs more interested in the subject matter are more willing to participate; however, during the focus group discussions, it became clear that HCPs with little experience in deprescribing of cardiometabolic medication also participated. One focus group included no GPs and one included no NPs. This could be a limitation because their absence might have made the discussion outcomes less representative. After conducting three focus groups with an overall balanced participation of HPCs, all subjects from the topic list had been discussed, whereas no new issues had emerged during the last focus group discussion.

\subsection{Implications for Research and Practice}

Although deprescribing of cardiometabolic drugs seems feasible, its implementation needs further support. We must focus on reducing the barriers and taking advantage of the enablers. Regarding the theme 'evidence and expertise', there are many points that require attention. Recently, the Dutch guideline Polypharmacy in elderly was supplemented 
by a section on deprescribing, which partially fills the information gap perceived by some HCPs by providing fact sheets [38]. Considerations and criteria have been summarized for deprescribing of several medication classes, including cardiometabolic medication. This supplement also includes more general recommendations for how to conduct deprescribing. Previously, it was found that the implementation of deprescribing guidelines may increase the self-efficacy of HCPs [39]. In addition, increasing awareness for the need of deprescribing is required. Reminder systems have been shown to increase deprescribing of glucose-lowering medication [40, 41]. Some HCPs may need additional training on how to communicate with patients and relatives when addressing deprescribing cardiometabolic medication. For some HCPs, it may be difficult to switch from stressing the need for using such medication towards explaining that the medication is no longer needed. HCPs can be supported with practical guidance, tailored education, and training, as was shown in a recent pilot study [42].

Some of the barriers associated with the theme 'beliefs and fears' could be addressed by making use of enablers, particularly by involving patients and their relatives in the decisionmaking process. HCPs and patients should openly talk about their beliefs and fears. A recent review showed that many primary care patients were willing to have their medication deprescribed when their doctor said it was possible [43]. This was also the case for older patients using cardiometabolic medication [44]. Shared decision making, monitoring the effects of deprescribing and having the option to restart were enablers for deprescribing cardiometabolic medication mentioned by not only HCPs but also by patients [45]. It is clear that these instruments should be an integral part of the deprescribing process.

Regarding the theme ' professional collaboration', our study illustrates that addressing only issues at the individual level of HCPs is probably not sufficient to bring about a shift from reactive deprescribing to proactive deprescribing. This shift largely depends on changes in the way in which care is provided. A multidisciplinary approach with agreements on the exchange of information between HCPs and a clear delegation of tasks should support the implementation of deprescribing as standard care.

Regarding barriers raised in the theme "context and resources', applying time-saving (digital) technology and tools to support a proactive and patient-centred approach is not only useful but is also urgently needed [23, 46]. Barriers regarding the environmental resources, such as a lack of time and inadequate financial compensation, can, to a certain extent, be addressed with more research on the cost effectiveness of implementing deprescribing.

\section{Conclusions}

HCPs recognize the importance of deprescribing cardiometabolic medication as a medical decision that needs to be based on evidence and sufficient patient information, and in close consultation with the patient. Primary care HCPs experienced several medication-specific barriers related to their knowledge, skills and fears. Tailored education and targeted training can adequately address these issues. Common general barriers and enablers identified by HCPs from all disciplines included the clear need for supportive evidence, a closer collaboration and improved communication between HCPs, involving patients and their relatives, and having sufficient time and resources to conduct appropriate deprescribing. A multidisciplinary approach is recommended to successfully accomplish the process of deprescribing. Within this approach, agreements about the roles and responsibilities of the different disciplines are needed.

Acknowledgements This research has been conducted with the support of an unconditional grant from the KNMP. The researchers are members of the DISCARDt (DeIntensifying CARdiovascular and Diabetes treatment) consortium, focusing on deprescribing and optimizing pharmacotherapy in older patients in The Netherlands.

\section{Declarations}

Funding An unconditional grant has been provided by The Royal Dutch Pharmacists Association (KNMP) in order to perform this research (grant no. PR18_0101).

Conflicts of interest/competing interests Jamila Abou, Stijn Crutzen, Vashti Tromp, Mette Heringa, Rob Van Marum, Petra Elders, Katja Taxis, Petra Denig, and Jacqueline Hugtenburg have no conflicts of interest.

Ethics approval The study was approved by the Medical Ethics Committee of the Amsterdam UMC, location VUmc (FWA00017598).

Consent to participate Written informed consent was obtained from participants prior to each focus group discussion.

Consent for publication Participants agreed with the use of anonymous citations in any publications.

Availability of data and material The datasets presented in this article are not readily available because this would be in conflict with the informed consent signed by the participants. Requests to access the datasets should be directed to the corresponding author (j.abou@ amsterdamumc.nl).

Code availability Not applicable.

Author contributions Study concept and design: JA, JH, PD, SC, KT. Acquisition of data: JA, JH, PD. Data analysis: JA, JH, PD, VT. Data interpretation: JA, JH, VT, SC. Manuscript drafting: JA. Manuscript critical revision and approval: All authors. 
Open Access This article is licensed under a Creative Commons Attribution-NonCommercial 4.0 International License, which permits any non-commercial use, sharing, adaptation, distribution and reproduction in any medium or format, as long as you give appropriate credit to the original author(s) and the source, provide a link to the Creative Commons licence, and indicate if changes were made. The images or other third party material in this article are included in the article's Creative Commons licence, unless indicated otherwise in a credit line to the material. If material is not included in the article's Creative Commons licence and your intended use is not permitted by statutory regulation or exceeds the permitted use, you will need to obtain permission directly from the copyright holder. To view a copy of this licence, visit $\mathrm{http} / / /$ creativecommons.org/licenses/by-nc/4.0/.

\section{References}

1. Maher RL, Hanlon JT, Hajjar ER. Clinical consequences of polypharmacy in elderly. Expert Opin Drug Saf. 2014;13:57-65.

2. Gnjidic D, Hilmer SN, Blyth FM, et al. Polypharmacy cutoff and outcomes: five or more medicines were used to identify community-dwelling older men at risk of different adverse outcomes. J Clin Epidemiol. 2012;65:989-95.

3. Wastesson JW, Morin L, Tan ECK, Johnell K. An update on the clinical consequences of polypharmacy in older adults: a narrative review. Expert Opin Drug Saf. 2018;17(12):1185-96.

4. Seidu S, Kunutsor SK, Topsever P, et al. Deintensification in older patients with type 2 diabetes: a systematic review of approaches, rates and outcomes. Diabetes Obes Metab. 2019;21:1668-79.

5. Cahir C, Bennett K, Teljeur C, Fahey T. Potentially inappropriate prescribing and adverse health outcomes in community dwelling older patients. Br J Clin Pharmacol. 2014;77:201-10.

6. Bruin-Huisman L, Abu-Hanna A, Van Weert HCPM, Beers E. Potentially inappropriate prescribing to older patients in primary care in the Netherlands: a retrospective longitudinal study. Age Ageing. 2017;46:614-9.

7. Krishnaswami A, Steinman MA, Goyal P, et al. Deprescribing in older adults with cardiovascular disease. Am Coll Cardiol. 2019;73:2584-95.

8. Farrell B, Black C, Thompson W, et al. Deprescribing antihyperglycemic agents in older patients: evidence-based clinical practice guideline. Can Fam Physician. 2017;63:832-43.

9. Scott IA, Hilmer SN, Le Couteur DG. Going beyond the guidelines in individualising the use of antihypertensive drugs in older patients. Drugs Aging. 2019;36:675-85.

10. Farrell B, Mangin D. Deprescribing is an essential part of good deprescribing. Am Fam Physician. 2019;99:7-9.

11. Scott IA, Hilmer SN, Reeve E, et al. Reducing inappropriate polypharmacy: the process of deprescribing. JAMA Intern Med. 2015;175:827-34.

12. Duncan P, Duerden M, Payne RA. Deprescribing: a primary care perspective. Eur J Hosp Pharm. 2017;24:37-42.

13. Page A, Clifford R, Potter K, Etherton-Beer C. A concept analysis of deprescribing medications in older people. J Pharm Pract Res. 2018;48:132-48

14. Reeve E, Gnjidic D, Long J, Hilmer S. A systematic review of the emerging definition of 'deprescribing' with network analysis: implications for future research and clinical practice. Br J Clin Pharmacol. 2015;80:1254-68.

15. Oktora MP, Kerr KP, Hak E, et al. Rates, determinants and success of implementing deprescribing in people with type 2 diabetes: a scoping review. Diabet Med. 2021;38: e14408.
16. Silverii GA, Caldini E, Dicembrini I, et al. Deprescription in elderly patients with type 2 diabetes mellitus. Diabetes Res Clin Pract. 2020;170: 108498.

17. Reeve E, Jordan V, Thompson W, et al. Withdrawal of antihypertensive drugs in older people. Cochrane Database Syst Rev. 2020;6(6): CD012572.

18. American Diabetes Association. 12. Older adults. Standards of care in diabetes-2021. Diabetes Care. 2021;44(Suppl. 1):S168-79.

19. Avery AJ. Rationalising medications through deprescribing. BMJ. 2019;364:I570.

20. Anderson K, Stowasser D, Freeman C, Scott I. Prescriber barriers and enablers to minimising potentially inappropriate medications in adults: a systematic review and thematic synthesis. BMJ Open. 2014;4: e006544.

21. Anderson K, Foster M, Freeman C, et al. Negotiating "unmeasurable harm and benefit": perspectives of general practitioners and consultant pharmacists on deprescribing in the primary care setting. Qual Health Res. 2017;27:1936-47.

22. Gillespie R, Mullan J, Harrison L. Deprescribing for older adults in Australia: factors influencing GPs. Aust J Prim Health. 2018;24:463-9.

23. Doherty AJ, Boland P, Reed J, et al. Barriers and facilitators to deprescribing in primary care: a systematic review. BJGP Open. 2020;4(3):bjgpopen20X101096.

24. Lau S, Lun P, Ang W, et al. Barriers to effective prescribing in older adults: applying the theoretical domains framework in the ambulatory setting - a scoping review. BMC Geriatr. 2020;20:459.

25. Turner JP, Edwards S, Stanners M, et al. What factors are important for deprescribing in Australian long-term care facilities? Perspectives of residents and health professionals. BMJ Open. 2016;6: e009781.

26. Goyal P, Anderson TA, Bernacki GM, et al. Physician perspectives on deprescribing cardiovascular medications for older adults. J Am Geriatr Soc. 2020;68:78-96.

27. Van Mil JWF, Tromp DFJ, Vos R, McElnay JC. Development of pharmaceutical care in The Netherlands: pharmacy's contemporary focus on the patient. J Am Pharm Assoc. 1999;39:395-401.

28. Costa FA, Scullin C, Al-Taani G, et al. Provision of pharmaceutical care by community pharmacists across Europe: is it developing and spreading? Eval Clin Pract. 2017;23:1336-47.

29. Atkins L, Francis J, Islam R, et al. A guide to using the theoretical domains framework of behaviour change to investigate implementation problems. Implement Sci. 2017;12:77.

30. Van Loenen T, Van den Berg MJ, Heinemann S, et al. Trends towards stronger primary care in three western European countries; 2006-2012. BMC Fam Pract. 2016;17:59.

31. Gerlach N, Michiels-Corsten M, Viniol A, et al. Professional roles of general practitioners, community pharmacists and specialist providers in collaborative medication deprescribing - a qualitative study. BMC Fam Pract. 2020;21:183.

32. D’Avanzo B, Agosti P, Reeve E, et al. Views of medical practitioners about deprescribing in older adults: findings from an Italian qualitative study. Maturitas. 2020;134:29-33.

33. Avery AJ, Rodgers S, Cantrill J. A pharmacist-led information technology intervention for medication errors (PINCER): a multicentre, cluster randomised, controlled trial and cost-effectiveness analysis. Lancet. 2012;379:1310-9.

34. Verdoorn S, Kwint H-F, Blom JW, et al. Effects of a clinical medication review focused on personal goals, quality of life, and health problems in older persons with polypharmacy: a randomised controlled trial (DREAMeR-study). PLOS Med. 2019;16: e1002798.

35. Anderson T, Goyal M, Marcum A. Implementing a proactive deprescribing approach to prevent adverse drug events. J Gen Intern Med. 2020;35:3694-6. 
36. Ailabouni NJ, Nishtala PS, Mangin D, Tordoff JM. General practitioners' insight into deprescribing for the multimorbid older individual: a qualitative study. Int J Clin Pract. 2016;70:261-76.

37. Jhangiani R, Tarry H, Stanger C. Principles of social psychology-1st international edition. Victoria, BC: BCcampus; 2014. https://opentextbc.ca/socialpsychology/. Accessed 14 Jun 2021.

38. Multidisciplinaire richtlijn Polyfarmacie bij ouderen [Multidisciplinary Guideline Polypharmacy in Elderly]. December 2020. https://richtlijnen.nhg.org/multidisciplinaire-richtlijnen/polyf armacie-bij-ouderen. Accessed 16 Apr 2021.

39. Farrell B, Richardson L, Raman-Wilms L, et al. Self-efficacy for deprescribing: a survey for health care professionals using evidence-based deprescribing guidelines. Res Soc Adm Pharm. 2018;14:18-25.

40. Wright SM, Hedin SC, McConnell M, et al. Using shared decision-making to address possible overtreatment in patients at high risk for hypoglycemia: The Veterans Health Administration's Choosing Wisely hypoglycemia safety initiative. Clin Diabetes. 2018;36:120-7.

41. Vimalananda VG, DeSotto K, Chen T, et al. A quality improvement program to reduce potential overtreatment of diabetes among veterans at high risk of hypoglycemia. Diabetes Spectr. 2017;30:211-6.

42. Crutzen S. Targeted and tailored pharmacist-led medication reviews: addressing deprescribing and hypoglycaemia in older people and people with type 2 diabetes [thesis]. University of Groningen; 2021. https://doi.org/10.33612/diss.192818811.

43. Weir KR, Ailabouni NJ, Schneider CR, et al. Consumer attitudes towards deprescribing: a systematic review and meta-analysis. J Gerontol A Biol Sci Med Sci. 2021. https://doi.org/10.1093/ gerona/glab222.

44. Crutzen S, Abou J, Smits SE, et al. Older people's attitudes towards deprescribing cardiometabolic medication. BMC Geriatr. 2021;21:366.

45. Crutzen S, Baas G, Abou J, et al. Barriers and enablers of older patients to deprescribing of cardiometabolic medication: a focus group study. Front Pharmacol. 2020;11:1268.

46. Ailabouni NJ, Nishtala PS, Mangin D, Tordoff JM. Challenges and enablers of deprescribing: a general practitioner perspective. PLoS ONE. 2016;11(4): e0151066.

\section{Authors and Affiliations}

\section{Jamila Abou ${ }^{1}\left[\right.$ ] Stijn Crutzen ${ }^{2} \cdot$ Vashti Tromp $^{1} \cdot$ Mette Heringa $^{3,4} \cdot$ Rob Van Marum $^{5,6} \cdot$ Petra Elders $^{7} \cdot$ Katja Taxis $^{8}$. Petra Denig ${ }^{2}$. Jacqueline Hugtenburg ${ }^{1}$}

1 Department of Clinical Pharmacology and Pharmacy, Amsterdam UMC, Location VUmc, Amsterdam, The Netherlands

2 Department of Clinical Pharmacy and Pharmacology, University Medical Center Groningen, University of Groningen, Groningen, The Netherlands

3 SIR Institute for Pharmacy Practice and Policy, Theda Mansholtstraat 5B, 2331 JE Leiden, The Netherlands

4 Division of Pharmacoepidemiology and Clinical Pharmacology, Utrecht Institute for Pharmaceutical Sciences, Utrecht University, Utrecht, The Netherlands
5 Department of Elderly Care Medicine, Amsterdam Public Health Research Institute, Amsterdam UMC (location VUmc), The Netherlands

6 Geriatric Department and Center for Clinical Pharmacology, Jeroen Bosch Hospital, 's-Hertogenbosch, The Netherlands

7 Department of General Practice, Amsterdam UMC, location VU, Amsterdam Public Health research institute, Amsterdam, The Netherlands

8 Unit of PharmacoTherapy, Epidemiology and Economics, Groningen Research Institute of Pharmacy, University of Groningen, Groningen, The Netherlands 